\title{
Etude biophysique et socio-économique du fruitier forestier Detarium senegalense J. F. Gmel. en Basse-Casamance (Thiobon, Bignona, Sénégal)
}

\author{
Ousmane DIATTA ${ }^{1,2 *}$, Sékouna DIATTA ${ }^{1}$, Ousmane NDIAYE ${ }^{2}$, Malick DIATTA $^{3}$ et \\ Daouda NGOM ${ }^{1}$
}

\author{
${ }^{1}$ Université Cheikh Anta Diop, FST, Département de Biologie Végétale, Dakar, Sénégal. \\ ${ }^{2}$ Institut Sénégalais de Recherches Agricoles, CRZ Dahra, Sénégal. \\ ${ }^{3}$ Centre Forestier de Recyclage, Thiès, Sénégal. \\ *Auteur correspondant ; E-mail : sadiousmane@hotmail.com
}

Received: 05-03-2021

Accepted: $14-08-2021$

Published: 31-08-2021

\section{RESUME}

Au Sénégal, la Basse-Casamance est une zone où la recherche a du terrain à explorer en raison de la situation de conflit qui y prévaut depuis de nombreuses années. C'est l'une des principales zones de production des fruits de Detarium senegalense, qui sont très importants pour la consommation humaine et pour le gain de revenus. L'objectif de cette étude est d'établir les caractéristiques biophysiques et socio-économiques de Detarium senegalense dans le peuplement ligneux du terroir villageois de Thiobon en Basse-Casamance. Pour y parvenir, des enquêtes ont d'abord été menées auprès de la population, puis 64 relevés de végétation ont été effectués sur des placettes de $2500 \mathrm{~m}^{2}$. Les résultats ont montré que la flore est constituée de 85 espèces relevant de 73 genres appartenant à 26 familles botaniques. Les familles des Fabaceae, Moraceae, Anacardiaceae et Apocynaceae sont les plus diversifiées. Les espèces les plus fréquentes sont Detarium senegalense (93,75\%), Parkia biglobosa (75\%), Cassia sieberiana (68,75\%), Dialium guineense (64,06\%), Anacardium occidentale et Piliostigma reticulatum (54,69\%), Terminalia macroptera et Uvaria chamae (50\%). La densité réelle est de 342 individus.ha ${ }^{-1}$, la surface terrière est de $18,07 \mathrm{~m}^{2}$.ha $\mathrm{a}^{-1}$ avec un taux de recouvrement de $81,49 \%$ et un taux de régénération de $81,47 \%$ pour le peuplement. Les résultats des enquêtes ont révélé que les principaux usages faits de Detarium senegalense sont l'autoconsommation, la vente des fruits et la pharmacopée. $71 \%$ de la population affirment que la tendance de la population de Detarium senegalense est progressive et $56 \%$ pensent que sa production augmente au cours des années. Les formes de régénération de l'espèce sont le semis naturel et le rejet de souche. Les fruits de Detarium senegalense sont vendus par comptage et par panier à des prix variant de 5 francs CFA (Communauté Financière Africaine) le fruit à 12000 francs CFA le panier. Cette étude permettra d'établir la situation actuelle des ressources forestières de la zone, mais également d'en améliorer la gestion en proposant des pistes d'amélioration des méthodes de gestion existantes.

(C) 2021 International Formulae Group. All rights reserved.

Mots clés : Detarium senegalense, caractérisation, socio-économie, Basse-Casamance. 


\title{
Biophysical and socio-economic study of the forest fruit tree Detarium senegalense J. F. Gmel. in Lower Casamance (Thiobon, Bignona, Senegal)
}

\begin{abstract}
In Senegal, Basse-Casamance is an area where research has to explore because of the conflict situation that has prevailed there for many years. It is one of the main production areas for Detarium senegalense fruits, which are very important for human consumption and for income generation. The objective of this study was to establish the biophysical and socioeconomic characteristics of Detarium senegalense in the woody stand of the village of Thiobon in Lower Casamance. To achieve this, surveys were first conducted among the population and then 64 vegetation surveys were carried out on $2500 \mathrm{~m}^{2}$ plots. The results showed that the flora consists of 85 species from 73 genera belonging to 26 botanical families. The families Fabaceae, Moraceae, Anacardiaceae and Apocynaceae are the most diversified. The most frequent species are Detarium senegalense (93.75\%), Parkia biglobosa (75\%), Cassia sieberiana (68.75\%), Dialium guineense (64.06\%), Anacardium occidentale and Piliostigma reticulatum (54.69\%), Terminalia macroptera and Uvaria chamae (50\%). The actual density is 342 individuals.ha-1, the basal area is $18.07 \mathrm{~m}^{2}$.ha- 1 with a cover rate of $81.49 \%$ and a regeneration rate of $81.47 \%$ for the stand. The results of the surveys revealed that the main uses of Detarium senegalense are selfconsumption, sale of fruits and pharmacopoeia. $71 \%$ of the population affirm that the population trend of Detarium senegalense is progressive and $56 \%$ think that its production increases during the years. The forms of regeneration of the species are natural seedling and stump rejection. The fruits of Detarium senegalense are sold by counter and by basket at prices ranging from 5 CFA francs (Communauté Financière Africaine) per fruit to 12,000 CFA francs per basket. This study will establish the current situation of forest resources in the area, but also improve management by proposing ways to improve existing management methods.
\end{abstract}

(C) 2021 International Formulae Group. All rights reserved.

Keywords: Detarium senegalense, characterization, socio-economy, Lower Casamance.

\section{INTRODUCTION}

En Afrique, notamment au Sénégal, les fruitiers forestiers jouent un rôle important dans la sécurité alimentaire des populations. Les produits de cueillette contribuent directement au bien-être nutritionnel des populations (Freiberger et al., 1998) et constituent des suppléments alimentaires importants pour améliorer la qualité de l'alimentation journalière des populations rurales (Sène, 2000 ; Glew et al., 2005). Les arbres en Afrique de l'ouest fournissent des apports alimentaires qui ne sont pas toujours assurés par la production agricole classique (Sène, 2000). En outre, ces fruitiers sont une source importante de revenus servant à l'achat de denrées de première nécessité (Diop, 2013). La Basse-Casamance reste à ce jour un terrain à explorer pour la recherche, en particulier forestière en comparaison à d'autres zones du Sénégal comme le Ferlo, le bassin arachidier et le Sine-Saloum. En fait, depuis l'éclatement du conflit dans les années 1980, il est devenu rare de trouver des études scientifiques faites en Basse-Casamance surtout concernant le domaine sensible des ressources forestières. Les seules données disponibles sont souvent celles des services des Eaux et Forêts. Cependant, avec la création de l'Université Assane Seck de Ziguinchor, les études scientifiques recommencent mais encore timidement.

Dans un tel contexte, il est important et opportun de mener des études qui visent à améliorer et à renforcer les connaissances actuelles sur l'état de ces fruitiers forestiers, vue leur importance. Cela devrait permettre d'en pérenniser la production par la proposition de méthodes de gestion plus efficaces des ressources forestières. C'est dans ce contexte que s'inscrit cette étude dont le but est d'établir les caractéristiques biophysiques et socioéconomiques de Detarium senegalense dans le peuplement ligneux du terroir villageois de Thiobon en Basse-Casamance. 


\section{MATERIEL ET METHODES}

\section{Site d'étude}

L'étude est menée dans le village de Thiobon situé sur l'axe routier dit Départementale 200 (Boucle du Blouf) entre les villages de Kartiack et de Mlomp. Administrativement, Thiobon appartient à la commune de Kartiack qui fait partie de l'arrondissement de Tendouck, du département de Bignona et de la région de Ziguinchor (Figure 1). La Basse-Casamance est caractérisée par une concentration importante d'eaux marines sur le continent et une dominance des zones basses correspondant à des dépôts de boues marines fixées par les mangroves. Ses parties les plus hautes correspondent à des affleurements de grès argileux (Niane, 1984). Sur le plan structural, le Blouf est une région qui se caractérise par de bas plateaux atteignant rarement dix mètres d'altitude, des plaines, de nombreuses vallées arrosées par une multitude de cours d'eau pour la plupart saisonniers (Service Départemental de l'Agriculture de Bignona, 2007 cité par Sano et Sonko, 2014). Dans la communauté rurale de Kartiack, il existe des sols ferralitiques (dans les plateaux), des sols peu évolués ou de transition (sur un glacis) et des vasières (sols de tanne) selon Diémé (2009). La Basse-Casamance se situe dans le domaine climatique sud-soudanien côtier marqué par l'alternance d'une longue saison sèche (novembre à mai) et d'une courte saison des pluies allant de juin à octobre (Badji, 2013). Les températures en période de chaleur varient entre $26{ }^{\circ} \mathrm{C}$ et $34{ }^{\circ} \mathrm{C}$ le matin dans la zone côtière et entre $28{ }^{\circ} \mathrm{C}$ et $36{ }^{\circ} \mathrm{C}$ dans la zone continentale. En période de froid, l'est est plus frais le matin et la nuit mais en début d'aprèsmidi les températures peuvent monter jusqu'à plus de $30{ }^{\circ} \mathrm{C}$ (Service Départemental du Développement Rural de Bignona, 2007 cité par Sano et Sonko 2014). Selon le Service Départemental des Eaux et Forêts, deux formations végétales y sont principalement rencontrées : la savane arborée à l'est et la forêt secondaire à l'ouest. Des espèces telles que Elaeis guineensis, Parkia biglobosa, Daniellia oliveri, Ceiba pentandra, Dialium guineense, Detarium senegalense etc. y sont rencontrées.
Les activités à Thiobon se résument entre autres à l'agriculture, l'élevage, la cueillette, la poterie, le maraichage, l'ostréiculture etc.

\section{Données dendrométriques}

La méthode des transects a été utilisée pour l'échantillonnage et la collecte des données des espèces ligneuses. Au total 17 transects ont été prédéfinis pour 64 placettes de $2500 \mathrm{~m}^{2}$. Les paramètres dendrométriques mesurés sont :

- La hauteur totale pour apprécier la distribution en fonction des classes de hauteur. Elle va du collet de l'arbre à la feuille ou la branche la plus au sommet,

- Le diamètre moyen du houppier pour évaluer le recouvrement aérien. Ce diamètre est obtenu en faisant la moyenne des diamètres est et ouest du houppier,

- La distance entre les individus des espèces trouvées dans les placettes pour calculer la densité théorique,

- Le diamètre du tronc à $0,30 \mathrm{~m}$ et à 1,30 $\mathrm{m}$ du sol pour établir le recouvrement basal et apprécier la distribution en fonction des classes de diamètre.

Afin d'établir les caractéristiques du peuplement ligneux, les paramètres suivants ont été calculés à partir de ces données :

Richesse et diversité floristiques : Ces paramètres permettent d'exprimer la biodiversité d'une communauté ou d'une biocénose à partir de sa composition en espèces, soit simplement en fonction de leur nombre (richesse), soit en tenant également compte de leurs effectifs (diversité) (Lacoste et Salanon, 2001). D'après Ozenda (2000), la richesse floristique est le nombre total d'espèces spontanées qu'il renferme.

Pour appréhender la diversité du peuplement, l'indice de diversité de Shannon-Weaver (H') a été calculé. Il mesure précisément la quantité moyenne d'information donnée par l'indication de l'espèce d'un individu de la collection. Il s'exprime en «bits». Selon le type de communauté, les valeurs de cet indice varient dans une fourchette approximative de 0 à 4,5 , rarement d'avantage (Frontier et PichodViale, 1998). Toutefois, pour un même nombre 
d'espèces, cette valeur sera d'autant plus élevée que leurs abondances respectives seront mieux équilibrées (Lacoste et Salanon, 2001). Sa formule est la suivante :

$$
\mathrm{H}^{\prime}=-\sum_{\mathrm{i}=1}^{\mathrm{s}} \frac{\mathrm{Ni}}{\mathrm{N}} \cdot \log 2 \frac{\mathrm{Ni}}{\mathrm{N}}
$$

Avec $H^{\prime}=$ indice de Shannon-Weaver ; $N i=$ effectif de l'espèce i et $N=$ effectif total des espèces

Cet indice est minimal si tous les individus du peuplement appartiennent à une seule et même espèce ; il est maximal quand tous les individus sont répartis d'une façon égale sur toutes les espèces (Frontier, 1983 cité par Ngom, 2014).

La surface terrière : Elle désigne la surface de l'arbre évaluée à la base du tronc (Ngom, 2014). Pour une espèce, la surface terrière correspond à la somme des surfaces terrières de ses individus. La surface terrière totale est la somme de toutes les surfaces terrières des espèces sur la surface échantillonnée.

Elle est exprimée en mètre carré par hectare $\left(\mathrm{m}^{2} \cdot \mathrm{ha}^{-1}\right)$. Elle est obtenue à partir de la formule suivante :

$$
S t=\frac{\sum \pi\left(\frac{d 0,3}{2}\right)^{2}}{S E}
$$

Avec $S_{t}=$ surface terrière ; $d_{0,3}=$ diamètre en $\mathrm{m}$ du tronc à $0,3 \mathrm{~m} ; S_{E}=$ surface de l'échantillon considéré en ha.

Le couvert ligneux : Il représente la surface de la couronne de l'arbre projetée verticalement au sol (Ngom, 2014) et indique la portion du sol couverte par le feuillage de l'arbre (Ndiaye, 2008). Il est exprimé en mètre carré par hectare $\left(\mathrm{m}^{2} \cdot \mathrm{ha}^{-1}\right)$ et est obtenu à partir de la formule suivante :

$C=\frac{\sum \pi\left(\frac{d \mathrm{mh}}{2}\right)^{2}}{S E}$

Avec $C=$ couvert ligneux $; d_{m h}=$ diamètre moyen du houppier en $\mathrm{m} ; S=$ surface de l'échantillon considéré en ha.

La densité: Elle désigne le nombre d'individus par unité de surface (Ngom, 2014). La densité d'une espèce correspond au nombre de pieds appartenant à l'espèce par unité de surface (Fofana, 2012). Elle s'exprime en individus par hectare $\left(\right.$ ind.ha $\left.^{-1}\right)$. Elle a été déterminée de deux façons : la densité observée et la densité théorique.

- La densité observée ou densité réelle est obtenue par le rapport de l'effectif total des individus par la surface échantillonnée.

Dob. $=\frac{\mathbf{N}}{\mathbf{S}}$

Avec $D o b .=$ densité observée $; N=$ effectif total dans l'échantillon considéré, $S=$ surface de l'échantillon en ha.

- La densité théorique : la distance moyenne entre les arbres sert à la calculer. Elle est obtenue par le rapport de la surface d'un hectare (en $\mathrm{m}^{2}$ ) sur le carré de la distance moyenne entre les arbres (Ngom, 2014).

Dth. $=\left(\frac{\mathbf{1 0 0}}{\mathbf{d m}}\right)^{2}$

Avec $D$ th $=$ densité théorique $; d m=$ distance moyenne entre les arbres.

La densité relative : Elle correspond à la proportion des individus d'une espèce par rapport aux individus de toutes les espèces. Elle est égale à l'effectif d'une espèce sur l'effectif total de l'échantillon multiplié par cent (Ngom, 2014).

$\mathrm{Dr}=\frac{\mathbf{N i}}{\mathbf{N}} \mathbf{X 1 0 0}$

Avec $D r=$ densité relative $; N i=$ effectif de l'espèce $\mathrm{i} ; \mathrm{N}=$ effectif total de l'échantillon.

La fréquence de présence $(F)$ : Elle renseigne sur la distribution d'une espèce dans un peuplement. Elle peut être exprimée en valeur absolue ou en pourcentage (Ngom, 2014). En pourcentage elle est donnée par la formule suivante :

$\mathbf{F}=\frac{\mathrm{Nri}}{\mathrm{Nr}} \mathrm{X100}$

Avec $F=$ fréquence de présence $; N r i=$ nombre de relevés où se retrouve l'espèce i; $N r=$ nombre total de relevés.

Le taux de régénération du peuplement (TRP): Il est donné par le rapport en pourcentage entre l'effectif total des jeunes plants et l'effectif total du peuplement (Poupon, 1980). 
$\mathrm{TRP}=\frac{\text { Effectif total des jeunes plants }}{\text { Effectif total du peuplement }} \mathrm{X} 100$ L'effectif total du peuplement regroupe aussi bien les jeunes plants que les plantes adultes.

\section{L'importance spécifique de régénération}

(ISR) : Elle obtenue à partir du rapport en pourcentage entre l'effectif des jeunes plants d'une espèce et l'effectif total des jeunes plants dénombrés (Akpo et Grouzis, 1996).

ISR $=\frac{\text { Effectif des jeunes plants d'une espèce }}{\text { Effectif total des jeunes plants dénombrés }} \mathrm{X} 100$

Sont considérés comme appartenant à la régénération, tous les sujets dont la circonférence est inférieure ou égale à $10 \mathrm{~cm}$ (Akpo et Grouzis, 1996 cités par Ngom, 2014).

\section{Données d'enquête}

Une enquête de type entretien a été menée auprès de quarante-huit personnes sur la base de l'échantillonnage boule de neige. C'est une technique qui permet à partir d'une personne ressource d'identifier et d'enquêter les personnes qu'il nous faut. Dans cette étude ce sont les personnes qui sont les plus en vue dans l'exploitation des fruits de Detarium senegalense et les personnes les plus âgées du village. Les données d'enquête ont été traitées avec le tableur Excel, le logiciel Sphinx.

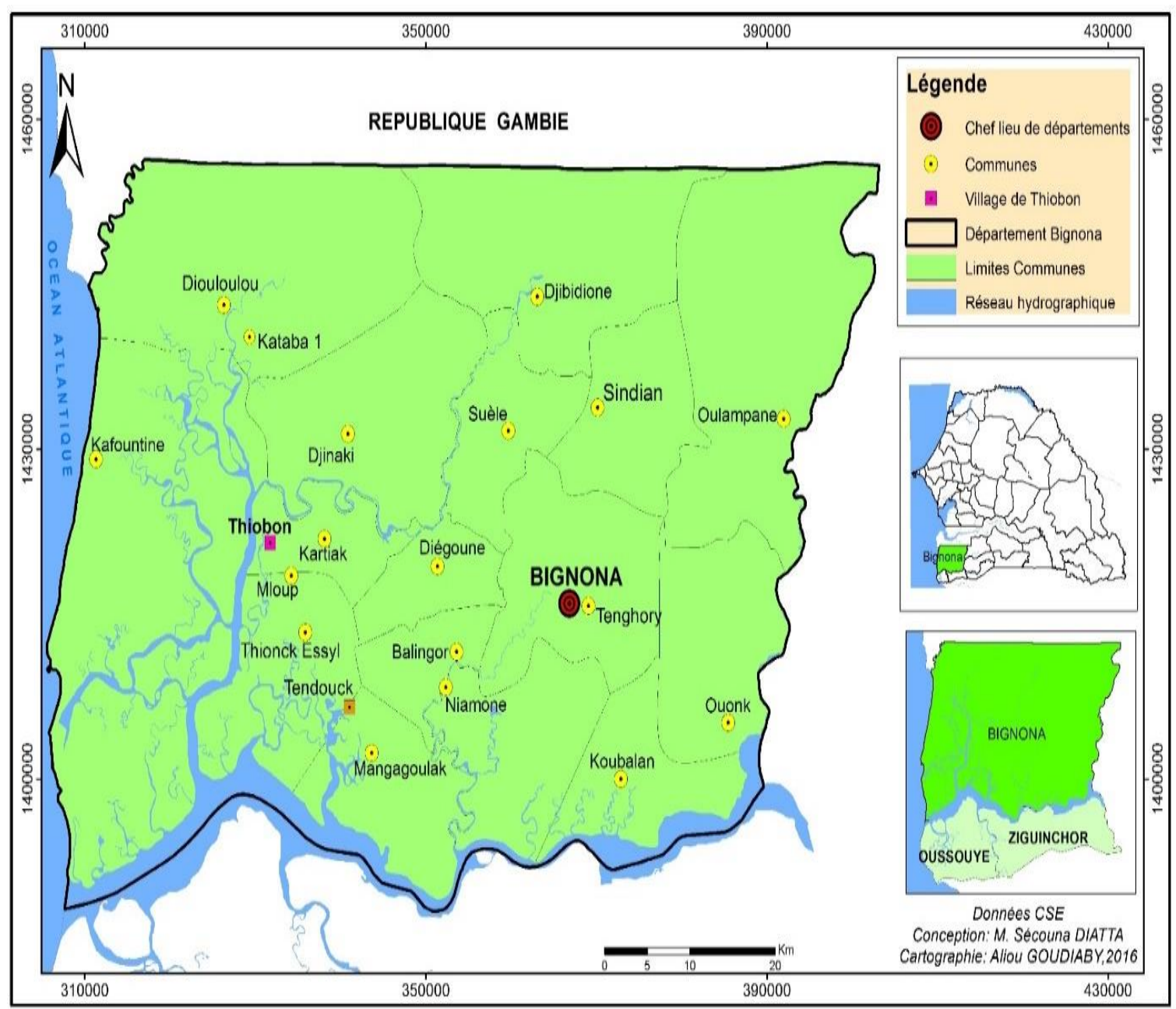

Figure 1 : Situation géographique et administrative de Thiobon. 


\section{RESULTATS \\ La richesse floristique dans le terroir de Thiobon}

Il a été dénombré sur le site 85 espèces relevant de 73 genres appartenant à 26 familles botaniques (Tableau 1) avec un indice de Shannon-Weaver égal à 4,7 bits. La famille des Fabaceae est la plus diversifiée avec 17 espèces.

\section{La répartition des ligneux selon la hauteur}

La Figure 2 présente la répartition des ligneux et des quatre espèces dominantes en classes de hauteurs.

La Figure 2 montre que les classes] 2-4] et] 4-6] renferment la majorité des individus $(58,55 \%)$ du peuplement et la classe] 2-4] renferme à elle seule $30,09 \%$ des ligneux. Detarium senegalense, Cassia sieberiana et Terminalia macroptera présentent la même allure que le peuplement avec comme classe modale la classe] 2-4]. Par contre Dialium guineense a comme classe modale la classe] 4$6]$.

\section{La répartition des ligneux selon le diamètre}

La répartition des ligneux et des quatre espèces dominantes est illustrée à la Figure 3.

Lorsqu'on considère la distribution des ligneux par rapport à leurs diamètres, la classe] 3,5-25] renferme l'essentiel des individus. La distribution des individus de Detarium senegalense suit la même tendance que le peuplement.

\section{La régénération du peuplement}

Le taux de régénération du peuplement est de $81,47 \%$. L'espèce qui régénère le plus est Guiera senegalensis. Detarium senegalense régénère par semis naturel et par rejet de souches avec un faible taux de régénération $(5,4 \%)$.

\section{Fréquence de présence, densité réelle, recouvrement et taux de régénération du peuplement}

Detarium senegalense fait partie des espèces les plus fréquentes dans le site. Le Tableau 2 donne quelques paramètres du peuplement ligneux et de Detarium senegalense.

\section{Les activités menées par la population de Thiobon}

A la Figure 4 sont présentées les activités principales menées par la population de Thiobon.

Il ressort de l'enquête que les principales activités menées dans le terroir de Thiobon sont l'agriculture et la cueillette (100\%) suivies de l'élevage $(93,75 \%)$.

\section{Les usages faits de Detarium senegalense}

Les différents usages que la population fait de Detarium senegalense sont présentés à la Figure 5. Les principaux usages en sont la consommation et la vente de ses fruits $(100 \%)$ mais l'espèce est aussi utilisée dans la pharmacopée locale $(37,5 \%)$.

La perception de l'évolution de la production fruitière de Detarium senegalense par la population

La Figure 6 fait état de la dynamique de la production de Detarium senegalense au fil des années. Dans le village, 56,25\% des personnes enquêtées affirment que la production augmente dans le temps et $27,08 \%$ disent qu'elle est en baisse.

\section{Les produits commercialisés par la population}

La Figure 7 indique les produits commercialisés dans le terroir de Thiobon. Elle montre que le premier produit commercialisé est le fruit de Detarium senegalense. Il est suivi des huîtres, de l'huile de palme, des fruits de Saba senegalensis, du poisson et de la pulpe d'Adansonia digitata.

\section{L'évolution du prix de vente des fruits de Detarium senegalense entre 1972-1975 et 2015}

Les Figures 8 et 9 présentent les prix de vente des fruits de Detarium senegalense. Dans les années 1972-1975, les prix les plus fréquents étaient 5 francs pour 5 fruits, 5 francs pour 4 fruits et 5 francs pour 3 fruits (Figure 8a). Actuellement (2015) les prix les plus fréquents sont 5 francs pour un fruit et 5 francs pour 2 fruits (Figure $8 b$ ). Ce qui témoigne de l'augmentation de la valeur marchande de ce produit forestier.

En 2015, en plus du comptage, les fruits sont aussi vendus par panier. Les prix du panier varient entre 6000 francs et 12000 francs (Figure 9). 
Tableau 1 : Liste des espèces recensées dans le terroir de Thiobon.

\begin{tabular}{|c|c|c|}
\hline Familles & Genres & Espèces \\
\hline \multirow[t]{8}{*}{ Anacardiaceae } & Anacardium & Anacardium occidentale $\mathrm{L}$. \\
\hline & Mangifera & Mangifera indica $\mathrm{L}$. \\
\hline & Lannea & Lannea acida A. Rich. \\
\hline & & Lannea nigritana (Scott.Elliot) Keay. \\
\hline & & Lannea $\operatorname{sp}(1)$ \\
\hline & & Lannea sp(2) \\
\hline & & Lannea velutina A.Rich. \\
\hline & Spondias & Spondias mombin L. \\
\hline \multirow[t]{2}{*}{ Annonaceae } & Annona & Annona senegalensis Pers. \\
\hline & Uvaria & Uvaria chamae P.Beauv. \\
\hline \multirow[t]{8}{*}{ Apocynaceae } & Baissea & Baissea multiflora A.DC. \\
\hline & Alstonia & Alstonia boonei De Wild. \\
\hline & Landolphia & Landolphia dulcis (Sabine) Pichon \\
\hline & Saba & Saba senegalensis (A.DC.) Pichon \\
\hline & Strophanthus & Strophanthus hispidus DG \\
\hline & & Strophanthus sarmentosus DC \\
\hline & Leptadenia & Leptadenia hastata (Pers.) Decne. \\
\hline & Landolphia & Landolphia heudelotii A.DC \\
\hline \multirow[t]{3}{*}{ Arecaceae } & Elaeis & Elaeis guineensis Jacq. \\
\hline & Cocos & Cocos nucifera $\mathrm{L}$. \\
\hline & Borassus & Borassus aethiopum Mart. \\
\hline \multirow[t]{2}{*}{ Bombacaceae } & Adansonia & Adansonia digitata $\mathrm{L}$. \\
\hline & Ceiba & Ceiba pentandra $\mathrm{L}$. \\
\hline Celastraceae & Salacia & Salacia senegalensis (Lam.) DC. \\
\hline \multirow[t]{2}{*}{ Chrysobalanaceae } & Parinari & Parinari excelsa Sabine \\
\hline & Neocarya & Neocarya macrophylla Sabine \\
\hline \multirow[t]{4}{*}{ Combretaceae } & Combretum & Combretum micranthum G.Don. \\
\hline & & Combretum paniculathum Subsp. \\
\hline & Guiera & Guiera senegalensis J.F.Gmel. \\
\hline & Terminalia & Terminalia macroptera Guill. \& Perr. \\
\hline \multirow[t]{2}{*}{ Connaraceae } & Byrsocarpus & Byrsocarpus coccineus auct. \\
\hline & Cnestis & Cnestis ferruginae DC. \\
\hline Dilleniaceae & Tetracera & Tetracera alnifolia Subsp.dinklagei (Gilg) Kubitzki \\
\hline \multirow[t]{4}{*}{ Euphorbiaceae } & Alchornea & $\begin{array}{l}\text { Alchornea cordifolia (Schumach. \& Thonn.) } \\
\text { Miill.Arg. }\end{array}$ \\
\hline & Anthostema & Anthostema senegalense A. Juss. \\
\hline & Jatropha & Jatropha curcas L. \\
\hline & Sapium & Sapium ellipticum (Hochst.) Pax \\
\hline Fabaceae & Afzelia & Afzelia africana Smith. \\
\hline
\end{tabular}




\begin{tabular}{|c|c|c|}
\hline & Piliostigma & Piliostigma reticulatum (DC.) Hochst \\
\hline & Faidherbia & Faidherbia albida (Delile) A.Chev. \\
\hline & Detarium & Detarium senegalense J.F.Gmel. \\
\hline & Parkia & Parkia biglobosa (Jacq.) R.Br. Ex G. Don. \\
\hline & Pterocarpus & Pterocarpus erinaceus Poir. \\
\hline & Andira & Andira inermis (W.Wright) DC. \\
\hline & Afrormosia & Afrormosia laxiflora (Benth) Harms \\
\hline & Dalbergia & Dalbergia rufa G.Don \\
\hline & Dialium & Dialium guineense Willd \\
\hline & Daniellia & Daniellia oliveri (Rolfe) Hutch.\& Dalziel \\
\hline & Erythrina & Erythrina crista galli L. \\
\hline & Cassia & Cassia sieberiana DC \\
\hline & Prosopis & Prosopis africana (Guill. \& Perr.) Taub. \\
\hline & Erythrophleum & Erythrophleum guineense auct. \\
\hline & Moghania & Moghania faginea (Guill.\& Perr.) kuntze \\
\hline & Tamarindus & Tamarindus indica $\mathrm{L}$. \\
\hline Gentianaceae & Anthocleista & Anthocleista nobilis G.Don \\
\hline Hypericaceae & Harungana & Harungana madagascariensis auct. \\
\hline Meliaceae & Khaya & Khaya senegalensis (Desr.) A. Juss. \\
\hline & Ekebergia & Ekebergia senegalensis A.Juss. \\
\hline & Azadirachta & Azadirachta indica A.Juss. \\
\hline Mimosaceae & Dichrostachys & 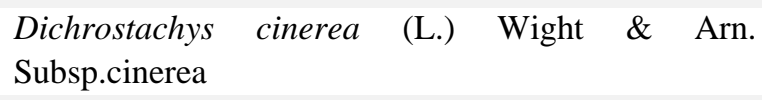 \\
\hline Moraceae & Antiaris & Antiaris africana Engl. \\
\hline & Morus & Morus mesozygia Stapf \\
\hline & Ficus & Ficus asperifolia Miq. \\
\hline & & Ficus dicranostyla Mildbr. \\
\hline & & Ficus leprieurii Miq. \\
\hline & & Ficus sp \\
\hline & & Ficus sur Forssk \\
\hline & & Ficus sycomorus L. \\
\hline & & Ficus vogelii (Miq.) Miq. \\
\hline Myrtaceae & Syzygium & Syzygium guineense (Willd.) DC \\
\hline Phyllanthaceae & Bridelia & Bridelia micrantha (Hochst.) Baill. \\
\hline Rubiaceae & Sarcocephalus & Sarcocephalus latifolius (Sm.) E.A.Bruce \\
\hline & Canthium & Canthium sp \\
\hline & Cephaelis & Cephaelis peduncularis Salisb. \\
\hline & Gardenia & Gardenia ternifolia Schumach.\& Thonn. \\
\hline Rutaceae & Citrus & Citrus lemon (L.) Burm.f. \\
\hline & Zanthhoxylum & $\begin{array}{l}\text { Zanthhoxylum zanthoxyloides (Lam.) Zepern. \& } \\
\text { Timler }\end{array}$ \\
\hline & Fagara & Fagara leprieurii auct. \\
\hline
\end{tabular}




\begin{tabular}{lll} 
Sapindaceae & Allophylus & Allophylus africanus P. Beauv. \\
& Aphania & Aphania senegalensis (Juss.ex Poir.) Radlk. \\
Sapotaceae & Malacantha & Malacantha alnifolia (Baker) Pierre \\
Simaroubaceae & Hannoa & Hannoa undulata (Gmill. \& Perr.) Planch. \\
Sterculiaceae & Cola & Cola cordifolia (Cav.) R.Br. \\
Verbenaceae & Vitex & Vitex doniana Sweet \\
& Tectona & Tectona grandis L.f. \\
\hline
\end{tabular}

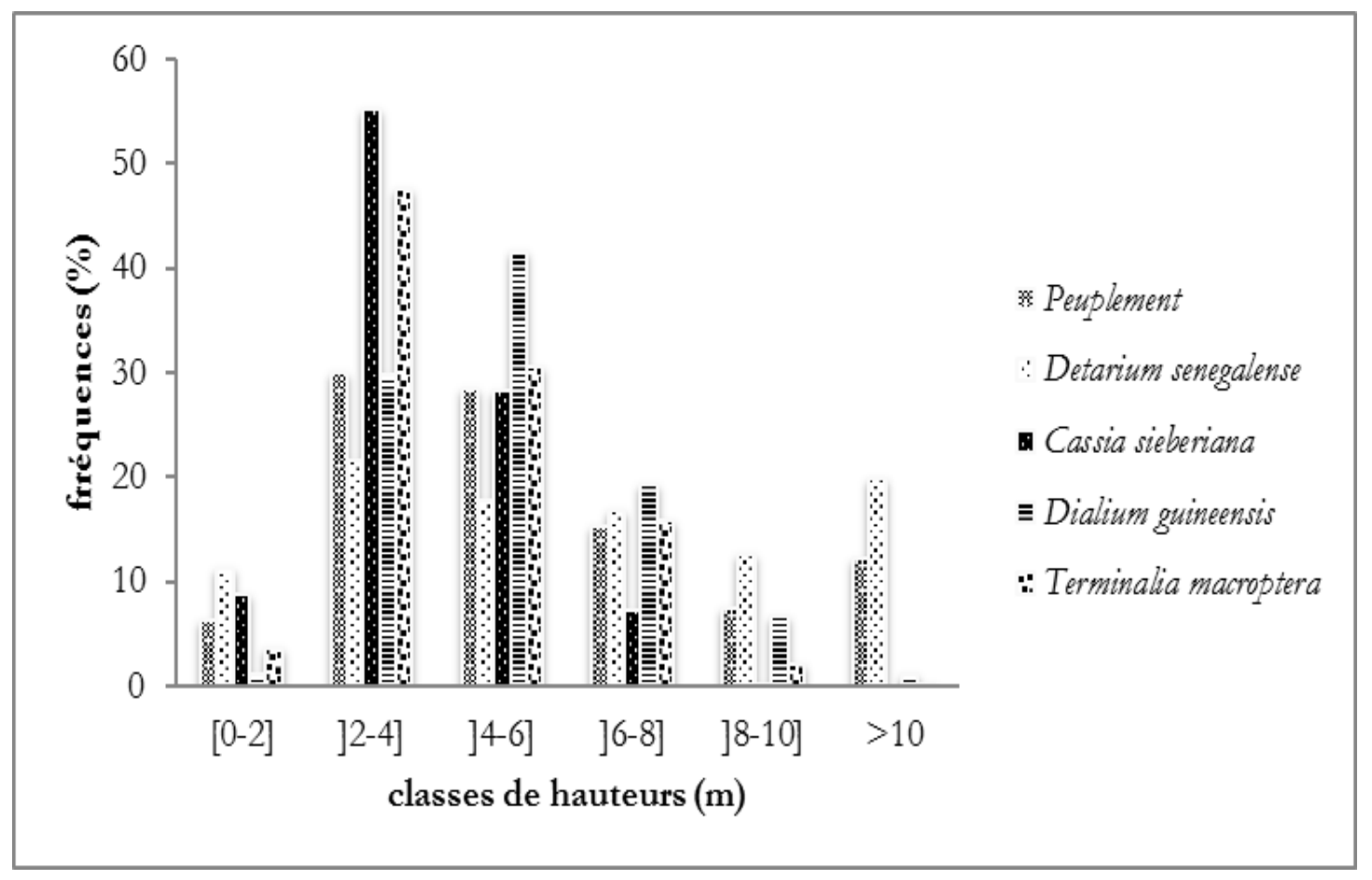

Figure 2 : Répartition des ligneux et des 4 espèces dominantes en fonction des classes de hauteurs.

Tableau 2 : Quelques paramètres du peuplement et de Detarium senegalense.

\begin{tabular}{lcc}
\hline Paramètres & Detarium senegalense & Peuplement \\
\hline Densité réelle $\left(\right.$ ind.ha $\left.^{-1}\right)$ & 58 & 342 \\
\hline Surface terrière $\left(\mathrm{m}^{2} \cdot \mathrm{ha}^{-1}\right)$ & 6,25 & 18,07 \\
\hline Recouvrement $\left(\mathrm{m}^{2} \cdot \mathrm{ha}^{-1}\right)$ & 2096,9 & 8149,175 \\
\hline Taux de régénération $(\%)$ & 5,4 & 81,47 \\
\hline
\end{tabular}




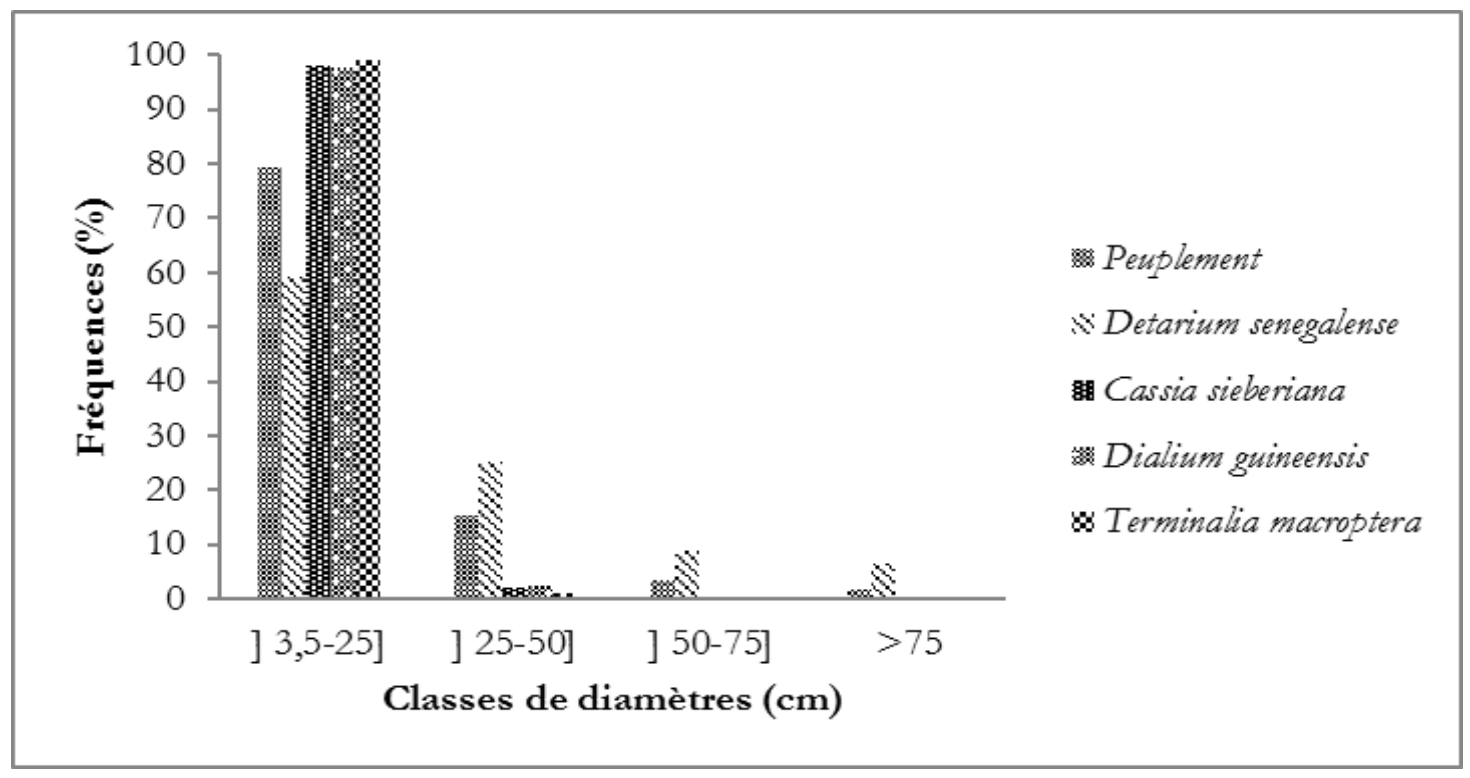

Figure 3 : Répartition du peuplement ligneux et des 4 espèces dominantes en classes de diamètre.

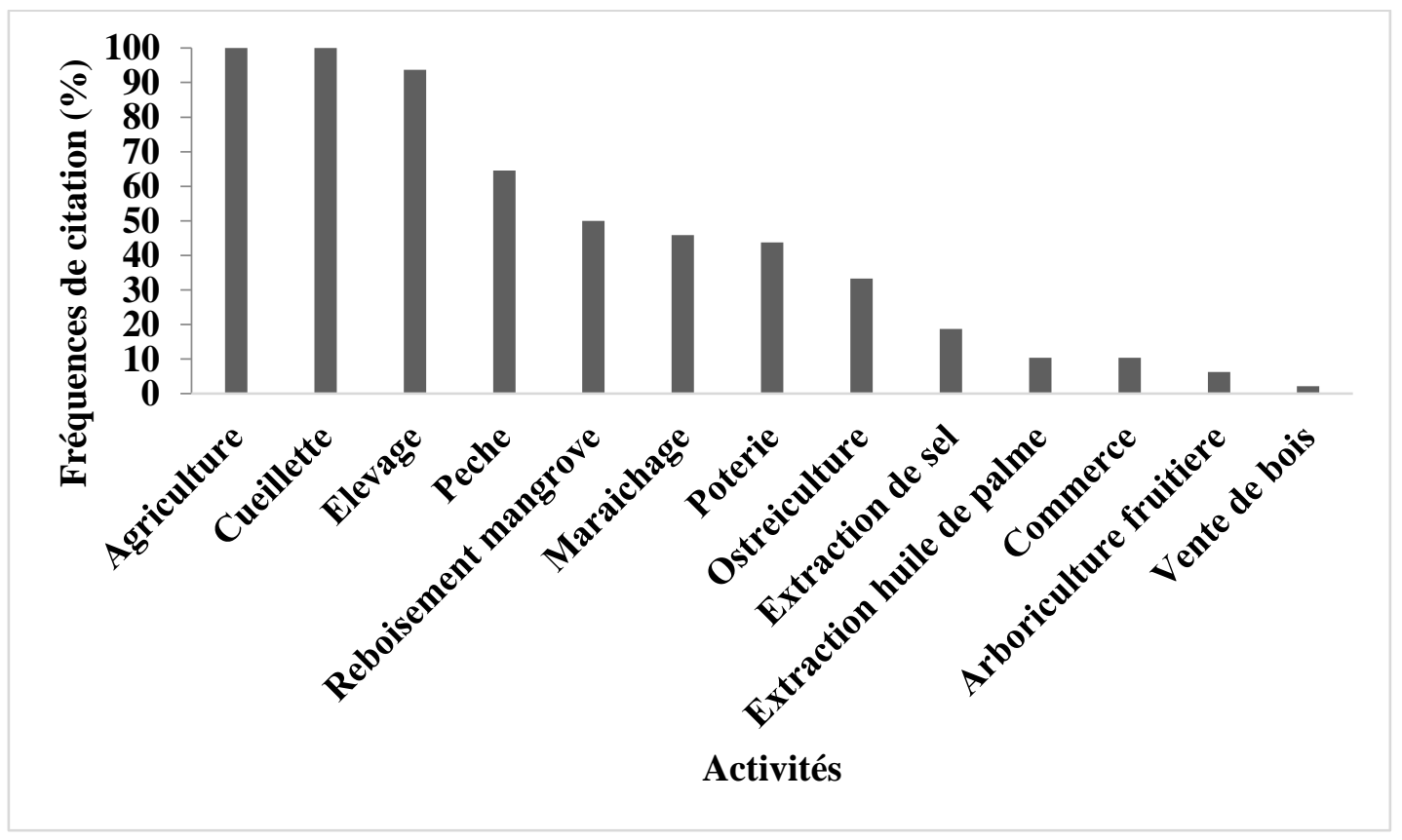

Figure 4 : Activités menées par la population de Thiobon. 


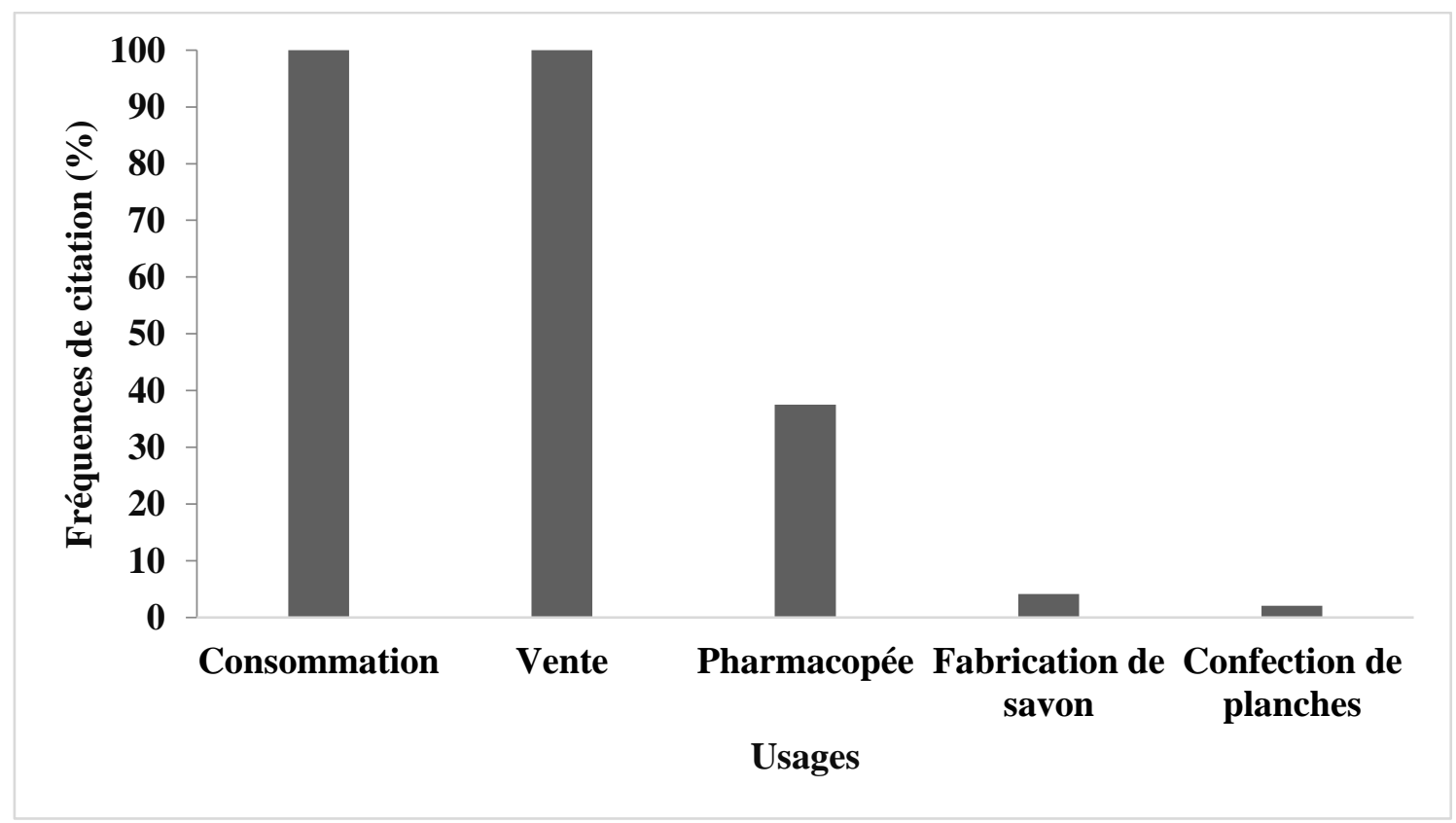

Figure 5 : Usages de Detarium senegalense dans le terroir villageois de Thiobon.

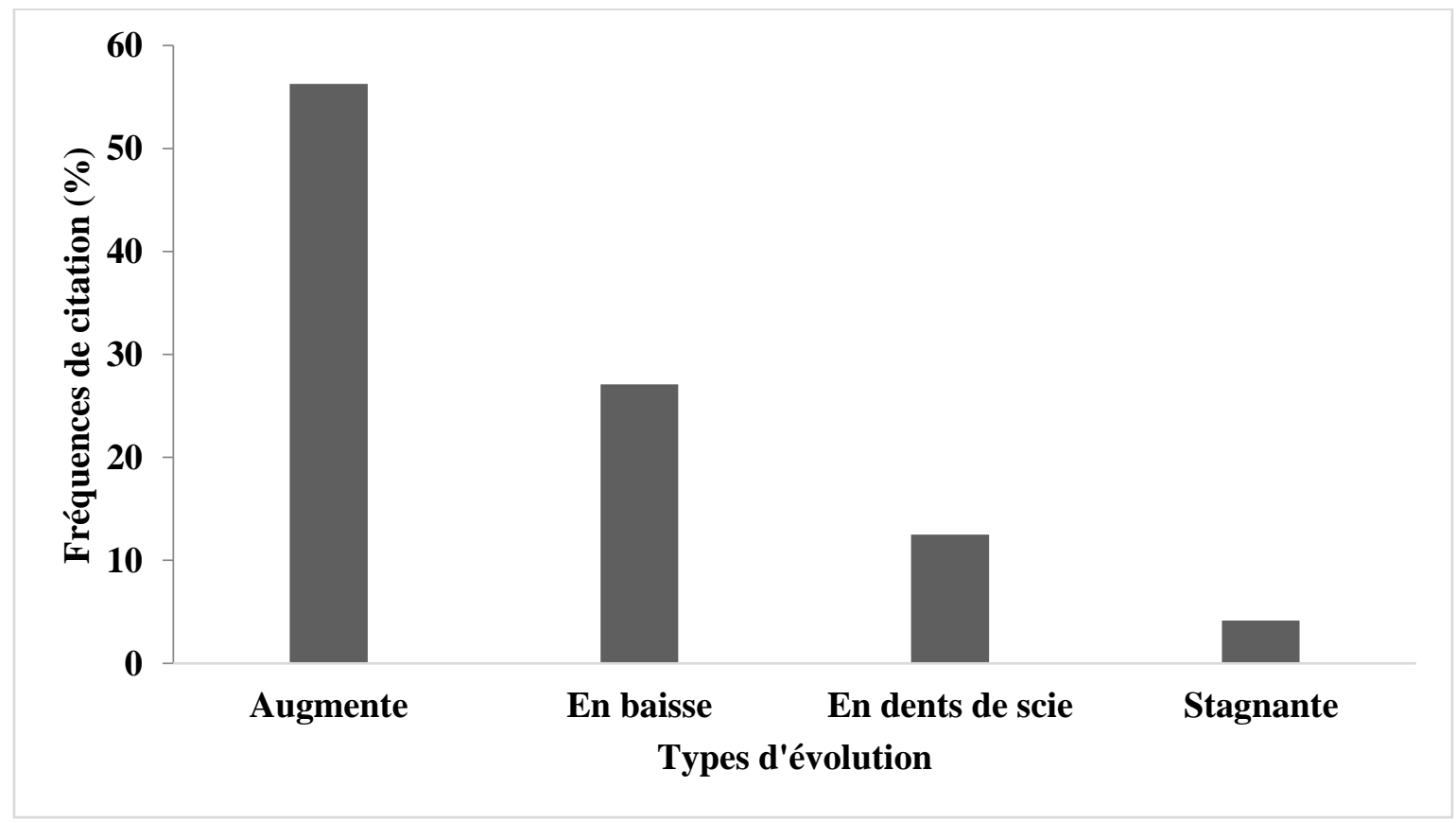

Figure 6 : Evolution de la production par la population. 


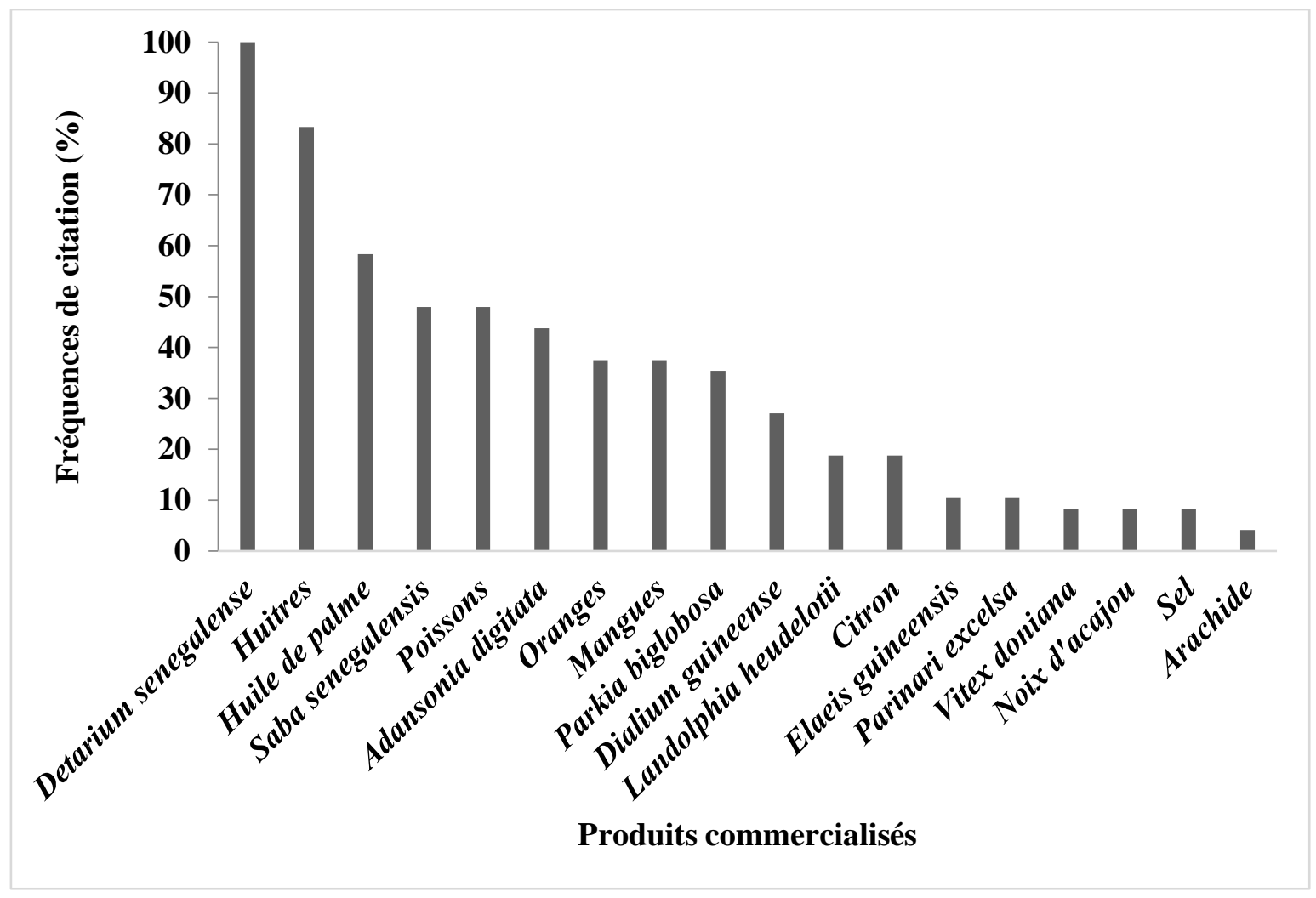

Figure 7 : Produits commercialisés dans le terroir villageois de Thiobon.

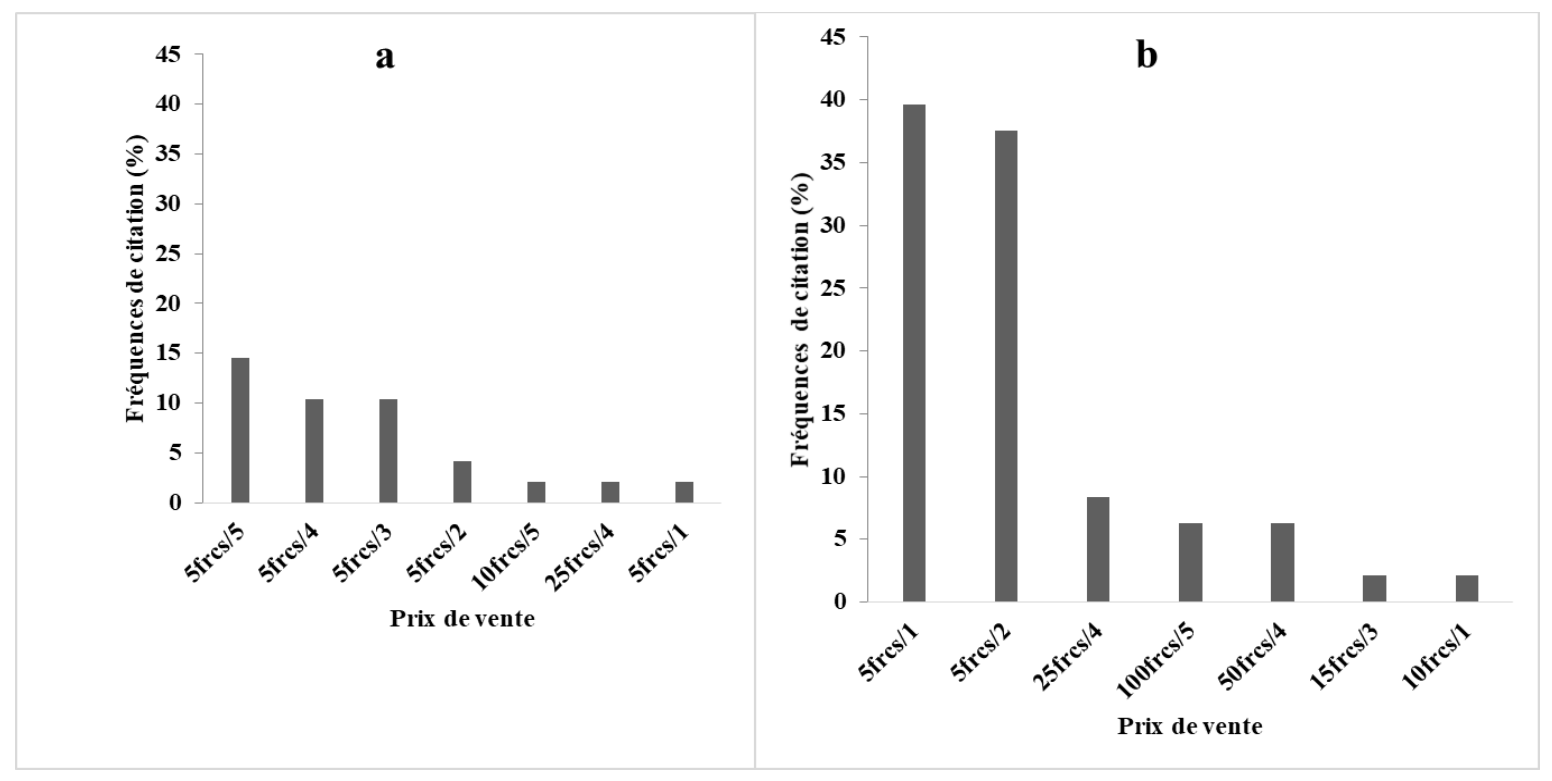

Figure 8 : Prix de vente au comptage des fruits de Detarium senegalense a) en 1972-1975 et b) 2015. 


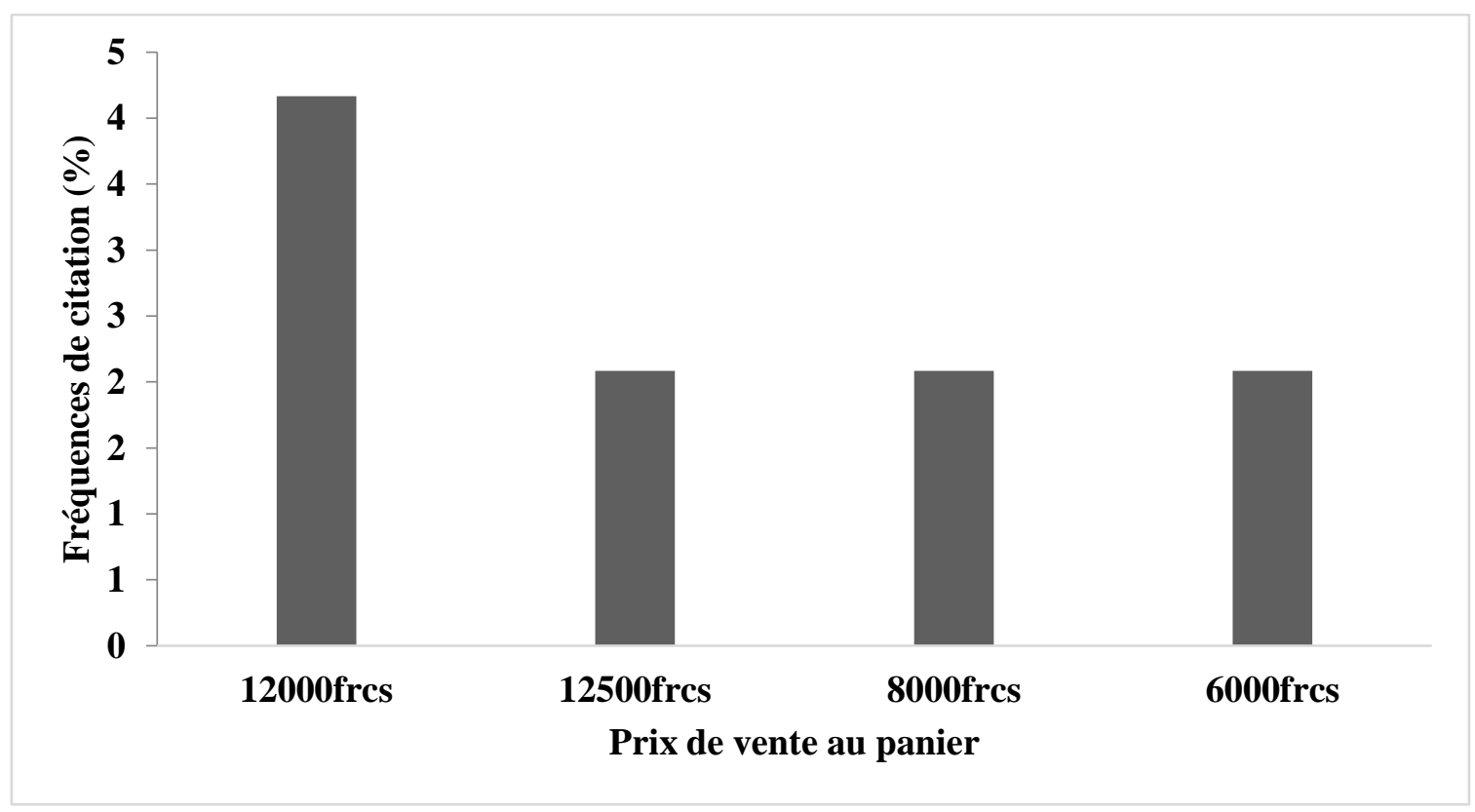

Figure 9 : Prix de vente au panier en 2015.

\section{DISCUSSION}

Le site présente une richesse spécifique de 85 espèces relevant de 73 genres appartenant à 26 familles botaniques. Ce résultat ne corrobore pas celui de Sano et Sonko (2014) qui ont recensé à Thiobon 14 espèces réparties en 14 genres et 11 familles et celui de Diop (1997) qui a recensé 48 espèces à Djimande, 47 espèces à Kagnarou et 39 espèces à Badiouré en Basse-Casamance. Sano et Sonko (2014) ont travaillé sur un petit nombre de placettes et Diop (1997) a travaillé uniquement sur des jachères, ce qui peut justifier la différence des résultats.

La famille des Fabaceae est la plus diversifiée contrairement aux résultats de Diop (1997) selon lesquels les Combretaceae dominent dans les jachères du département de Bignona. La valeur de l'indice de ShannonWeaver est de 4,7 bits dans la zone. Ngom et al. (2018) ont trouvé un indice de ShannonWeaver égal à 2,93 bits dans les palmerais de la commune de Kabiline frontalière de Thiobon.

La distribution des ligneux en classe de diamètre et de hauteur montre que la population de Detarium senegalense est jeune et dominée par les arbustes. Un résultat qui est confirmé par la population locale qui affirme que la population de l'espèce s'y renouvelle au fur et à mesure.

Le taux de régénération du peuplement est de $81,47 \%$. L'espèce qui régénère le plus est Guiera senegalensis. Detarium senegalense régénère par semis naturel et par rejet de souches avec un faible taux de régénération $(5,4 \%)$. Ce résultat confirme Taîta (2003) et Soumana et al. (2010) selon qui, les espèces dont les fruits et/ou les graines sont ramassés et commercialisés rencontrent des problèmes de régénération. Le ramassage des fruits destinés à la commercialisation serait donc l'explication à ce faible taux de régénération. Cependant ce faible taux de régénération de Detarium senegalense pourrait à la longue constituer un danger pour sa pérennité. L'espèce n'étant pas domestiquée dans la zone, la seule voie de régénération reste à ce jour la régénération naturelle qui est menacée par le ramassage des fruits.

Detarium senegalense fait partie des espèces les plus fréquentes dans le site. Diop 
(1997) a rapporté que cette espèce faisait partie des mieux représentées dans les jachères du département de Bignona. Le fait que cette espèce figure parmi les mieux représentées à l'état adulte alors que son taux de régénération est des plus faibles est assez ambiguë. Quand on regarde l'évolution des prix de vente des fruits, on se rend compte que dans les années 1975, la valeur marchande des fruits de Detarium senegalense n'était pas si élevée. Cependant, avec l'augmentation de cette valeur marchande, leur commerce a pris plus d'ampleur. Cela a pour conséquence de laisser sur place moins de graines pour permettre une bonne régénération des individus de l'espèce. Ceci peut constituer une piste vers la compréhension de cette situation qui semble contradictoire.

La plus grande part de la production fruitière de Detarium senegalense est destinée à la consommation et à la vente. D'autres produits tels que les huîtres, l'huile de palme, les fruits de Saba senegalensis, le poisson et la pulpe d'Adansonia digitata sont également commercialisés. Detarium sengalense est aussi utilisée dans la pharmacopée. En effet, une des caractéristiques des milieux ruraux reste la consommation des produits de cueillette. Pour diversifier leur consommation, une partie de ces produits de cueillette est souvent commercialisée afin de pouvoir acquérir des produits manufacturés.

La production fruitière de l'espèce serait en perpétuelle augmentation au fil des années, en partie grâce des initiatives locales comme la protection des individus jeunes comme adultes de l'espèce. Au cours des années, la valeur marchande des fruits de Detarium senegalense a augmenté comme en témoigne l'évolution des prix de vente. Ayant pris conscience de l'importance de cette espèce dans leur alimentation et le commerce, la population a érigé des règles pour en tirer le maximum de bénéfice. Cette situation permet de se rendre compte qu'une des choses les plus importantes dans la gestion participative des ressources forestières est d'arriver à faire prendre conscience la population de l'importance des ressources en question.

\section{Conclusion}

Ce travail a permis d'établir les caractéristiques biophysiques et socioéconomiques de Detarium senegalense dans le terroir villageois de Thiobon. Il a montré que le site est très riche et varié d'un point de vue botanique. Le peuplement est arbustif, jeune et stable. Detarium senegalense est très bien représentée à l'état adulte mais son taux de régénération est faible à cause du ramassage de ses fruits à des fins commerciales. Cette espèce est en fait au cœur de l'activité commerciale de la population de Thiobon. Ses usages dépassent la seule consommation et la commercialisation de ses fruits. Si les usages comme la fabrication de savon et de planche sont d'une grande ampleur, cela pourrait porter préjudice à la pérennité de l'espèce. Ce fruitier est d'autant plus important qu'au-delà des apports nutritionnels, la vente de ses fruits permet d'avoir des revenus pour acheter d'autres denrées. Des initiatives de gestion et d'organisation de la cueillette sont entreprises par la population elle-même. En complément, des techniques de gestion efficaces de la régénération devraient être mises en œuvre pour pérenniser la bonne présence de l'espèce au stade adulte et la production de fruits. L'abattage des individus de cette espèce pour son bois doit également faire l'objet d'une attention particulière. La zone étant éloignée des centres villes comme Bignona et Ziguinchor, il serait très bénéfique d'avoir une usine de transformation et de conditionnement des fruits au niveau local.

\section{CONFLIT D'INTERETS}

Les auteurs déclarent qu'ils n'ont pas de conflit d'intérêts.

\section{CONTRIBUTIONS DES AUTEURS}

SD, MD et OD ont conçu l'étude. OD a collecté et traité les données, et a rédigé la première version du manuscrit. $\mathrm{SD}, \mathrm{DN}$ et $\mathrm{ON}$ ont fait une lecture critique du document. 


\section{REFERENCES}

Akpo LE, Grouzis M. 1996. Influence du couvert ligneux sur la régénération de quelques espèces ligneuses sahéliennes (nord du Sénégal, Afrique occidentale). Webbia 50(2): 247-263. DOI : 10.1080/00837792.1996.10670605

Badji T. 2013. La variabilité pluviométrique et ses incidences sur la riziculture en basse Casamance : cas de la commune de Diouloulou. Mémoire de master2 option : ressources, environnement et développement (RED). FST, UCAD, 145 p.

Berhaut J. 1967. Flore du Sénégal (2 ${ }^{\mathrm{ème}}$ édn). Clairafrique : Dakar, Sénégal ; 485 p.

Berhaut J. 1971. Flore Illustrée du Sénégal, Dicotylédones (tome I), Acanthacées à Avicenniacées. Sénégal. Gouvernement du Sénégal Ministère du développement rural direction des Eaux et Forêts, 695 p.

Berhaut J. 1974. Flore Illustrée du Sénégal, Dicotylédones, tome II, Balanophoracées à composées. Sénégal. Gouvernement du Sénégal Ministère du développement rural direction des Eaux et Forêts, 695 p.

Berhaut J. 1975. Flore Illustrée du Sénégal, Dicotylédones, tome III, Connaracées à Euphorbiacées. Sénégal. Gouvernement du Sénégal Ministère du développement rural direction des Eaux et Forêts, 634 p.

Berhaut J. 1975. Flore Illustrée du Sénégal, Dicotylédones, tome IV, Ficoidées à Légumineuses. Sénégal. Gouvernement du Sénégal Ministère du développement rural direction des Eaux et Forêts, 625 p.

Berhaut J. 1976. Flore Illustrée du Sénégal, Dicotylédones, tome $V$, Légumineuses Papillionacées. Sénégal. Gouvernement du Sénégal Ministère du développement rural direction des Eaux et Forêts, 658 p.

Berhaut J. 1979. Flore Illustrée du Sénégal, Dicotylédones, tome VI, Linacées à Nymphéacées. Sénégal. Gouvernement du Sénégal Ministère du développement rural direction des Eaux et Forêts, 658 p.

Diémé S. 2010. Impacts de la dégradation des sols dans la communauté rurale de
Kartiack. Mémoire de maitrise. FLSH, UCAD, $108 \mathrm{p}$.

Diop N, 2013. Caractérisation du ditax (Detarium senegalense G.F.Gmel) et étude de sa transformation en nectar. Thèse de doctorat en systèmes énergétiques et environnement. ESP, UCAD, $165 \mathrm{p}$.

Diop G. 1997. Contribution à la connaissance des potentialités des jachères en espèces forestières ligneuses alimentaires et en espèces forestières ligneuses à usage médicinale dans le Département de Bignona Sénégal. Mémoire d'étude : (Formation Ingénieur d'Agronomie). ISRA, $90 \mathrm{p}$.

Fofana MAD. 2012. Caractéristiques de Maerua crassifolia forsk, ligneux fourrager, dans la réserve sylvopastorale de Sogobe, au Ferlo. Mémoire de master en Agroforesterie, Ecologie et Adaptation. FST, UCAD, 49 p.

Freiberger CE, Vanderjagt DJ, Pastuszyn A, Glew RS, Mounkaila G, Millson M, Glew RH. 1998. Nutrient content of the edible leaves of seven wild plants from Niger. Plant Foods for Human Nutrition, 53: 5769. DOI : 10.1023/A:1008080508028

Frontier F, Pichod-viale D. 1998. Ecosystèmes: Structure-Fonctionnement-Evolution (2 $2^{\mathrm{ème}}$ édn). Dunod: Paris, France; 447 p.

Glew RS, Dorothy J, Chuang LT, Huang YS, Millson M, Glew RH. 2005. Nutrient content of four edible wild plants from West Africa. Plant Foods Hum. Nutr., 60: 187-193.

Lacoste A, Salanon R. 2001. Eléments de Biogéographie et d'Ecologie (2 ${ }^{\mathrm{m} e}$ édn). Nathan/HER ; 300 p. DOI : 10.1007/S11130-005-8616-0

Lebrun JP, Stork AL. 1991. Enumération des Plantes à Fleurs d'Afrique Tropicale. Edition des Conservatoire et Jardin Botaniques : Genève ; 249 p.

Mané BS. 2010. L'impact du changement climatique sur l'environnement biophysique et socio-économique dans la communauté rurale de Niaguis (région de Ziguinchor). Mémoire de maitrise. FLSH, UCAD, $94 \mathrm{p}$. 
Ndiaye I. 2008. Flore et végétation ligneuses du terroir de Katane dans la réserve de faune du Ferlo-nord. Mémoire de DEA en Biologie végétale. FST, UCAD, 21 p.

Ngom D. 2014. Biodiversité et Services Ecosystémiques dans les Réserves de Biosphère : Réserve de Biosphère du Ferlo en Afrique de l'Ouest. Presses Académiques Francophones ; 184 p.

Ngom D, Camara B, Gomis ZD, Sagna B. 2018. Cortège floristique, paramètres structuraux et indicateurs d'anthropisation des parcs agroforestiers à Elaeis guineensis Jacq. en Basse Casamance, Sénégal. Journal of Animal and Plant Sciences, 36 (3) : 5919-5932.

Niane AB. 1984. Etudes cartographiques et agro-pédologiques des sols de plateau de Basse-Casamance. Mémoire de stage, CRA, ISRA, $111 \mathrm{p}$.

Ozenda P. 2000. Les Végétaux : Organisation et Diversité Biologique (2 $2^{\text {ème }}$ édn). Dunod : Paris, France ; 516 p.

Poupon H. 1980. Structure et dynamique de la strate ligneuse d'une steppe sahélienne au nord du Sénégal. ORSTOM éd. (Etudes \& Thèses), Paris : 307 p.
Ramade F. 2009. Eléments d'Ecologie : Ecologie Fondamentale (4 ${ }^{\text {ème }}$ édn). Dunod : Paris, France ; 689 p.

Sano L, Sonko I. 2014. Caractérisation biophysique et socioéconomique des parcs à Detarium senegalense J.F.Gmel. dans le terroir villageois de Thiobon (Département de Bignona). Mémoire de Licence en Agroforesterie. UFR S\&T, UASZ, $39 \mathrm{p}$.

Sène EH. 2000. Forêts et sécurité alimentaire en Afrique. La place de la foresterie dans le Programme spécial pour la sécurité alimentaire de la FAO. In Forêts, Sécurité Alimentaire et Moyens de Subsistance Durables. FAO (eds.).

Soumana D, Rabi C, Mahamane A, N'da DH, Saadou M. 2010. État actuel de dégradation des populations de quatre espèces ligneuses fruitières en zone Sahélo soudanienne du Niger : Réserve Totale de Faune de Tamou. Rev. Ivoir. Sci. Technol., 16: 191-210.

Taita P. 2003. Use of woody plants by locals in Mare aux Hippopotames Biosphère Réserve in western Burkina Faso. Biodiversity and Conservation, 12 : 1205 1217. DOI : $10.1023 / \mathrm{A}: 1023045316329$. 